\title{
Pseudoaneurisma de la arteria femoral profunda secundario a trauma penetrante. Reporte de caso
}

\author{
Héctor H. Romero-Garza ${ }^{1}$, Alan Fuentes-Ortiz ${ }^{1}$, \\ Rogelio Salinas-Domínguez ${ }^{1}$ y Gerardo E. Muñoz-Maldonado ${ }^{1}$
}

\section{Deep femoral artery pseudoaneurysm secondary to penetrating trauma. Case report}

\begin{abstract}
Aim: The aim of this paper is to present a case of a deep femoral artery pseudoaneurysm secondary to a penetrating trauma in the left thigh, its management and a literature review. Clinical Case: 21-year-old male referred to the emergency department of our institution 5 months after he was injured with a knife on his left thigh, with severe local swelling, local pain, and difficulty to the mobilization of the left leg. A $2.3 \mathrm{~cm}$ x $2.1 \mathrm{~cm}$ x $2.7 \mathrm{~cm}$ deep femoral artery pseudoaneurysm was diagnosed with a $13.5 \mathrm{~cm} \times 12.6 \mathrm{~cm} \times$ $23 \mathrm{~cm}$ adjacent hematoma. Endovascular exclusion was made with coils and evacuation of the hematoma with open surgery. Results: The patient was discharged on the third day postop without pain and walking with antibiotics and follow-up to a month did not reveal any complications. Discusion and Conclusion: Exclusion with coils is an adequate management in deep femoral pseudoaneurysms that facilitates the evacuation of the hematoma lowering the risk of bleeding.

Key words: pseudoaneurysm; coils; hematoma; deep femoral artery.
\end{abstract}

\section{Resumen}

Objetivo: El objetivo del trabajo es presentar una opción terapéutica adecuada para los pseudoaneurismas de la femoral profunda secundarios a trauma penetrante, así como realizar una revisión de la literatura sobre el manejo en estas patologías. Caso clínico: Paciente masculino de 21 años quien 5 meses previos a su valoración sufre una herida por arma punzocortante en el muslo izquierdo, desarrollando aumento de volumen el sitio de la lesión, dolor y limitación al movimiento. Se diagnostica un pseudoaneurisma de la arteria femoral profunda de 2,3 cm x 2,1 cm x 2,7 cm y un hematoma adyacente de 13,5 cm x $12,6 \mathrm{~cm} \times$ $23 \mathrm{~cm}$. Se realiza exclusión del pseudoaneurisma mediante cirugía endovascular con coils, posteriormente se evacúa el hematoma adyacente. Resultados: El paciente egresa al tercer día posoperatorio con mejoría de la sintomatología, antibioticoterapia y analgesia. Discusión y Conclusión: En el caso presentado la exclusión del pseudoaneurisma mediante coils facilitó el control de éste y la evacuación del hematoma adyacente, disminuyendo el riesgo de sangrado. Por lo que consideramos adecuada esta conducta terapéutica en pseudoaneurismas de la femoral profunda.

Palabras clave: pseudoaneurisma; coils; hematoma; arteria femoral profunda.

\section{Introducción}

Los pseudoaneurismas de la arteria femoral profunda son entidades poco comunes con una incidencia reportada del $2 \%$ de las lesiones arteriales periféricas, siendo la causa más frecuente la iatrogénica, como en procedimientos vasculares y ortopédicos, seguida por el trauma penetrante ${ }^{1}$. Un pseudoaneu- risma ocurre al presentarse un defecto en una o más capas de la pared arterial que permite la salida de sangre de la luz, formando un saco localizado con flujo debajo de la adventicia o en los tejidos circundantes $^{1,2}$. El estándar de oro de tratamiento en los pseudoaneurismas es el manejo quirúrgico ya sea ligando el cuello del pseudoaneurisma y evacuando el hematoma o realizando una angioplastia con parche ${ }^{2}$.
"Hospital Universitario "Dr. José Eleuterio González" Universidad Autónoma de Nuevo León. México.

Recibido 2019-09-29 y aceptado 2020-01-13

Correspondencia a:

Dr. Héctor H. Romero-Garza hromero.garza@gmail.com 


\section{CASOS CLÍNICOS}

\section{Caso clínico}

Se presenta el caso de un paciente de sexo masculino de 21 años sin antecedentes médicos, quien 5 meses previos a su valoración es herido por arma

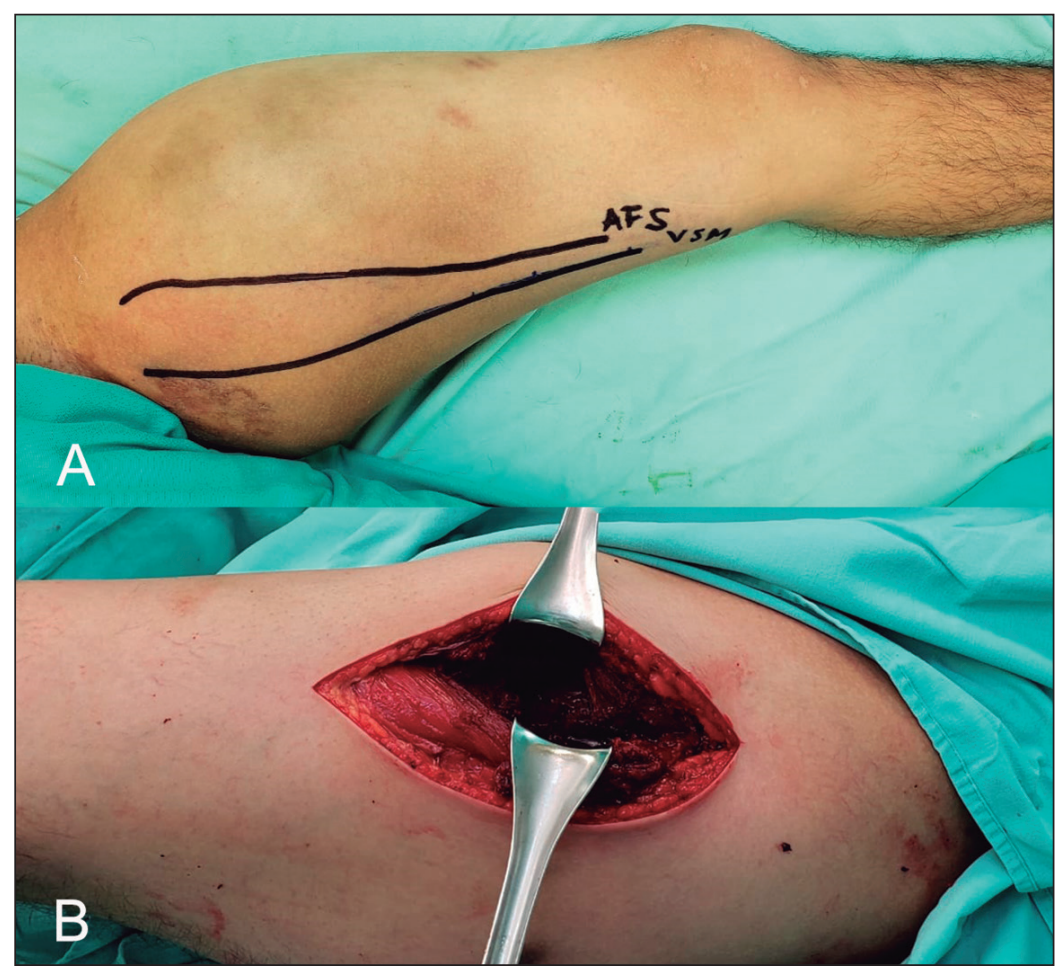

Figura 1. A) Imagen clínica de la extremidad inferior izquierda, con aumento de volumen. Se observa marcaje por ultrasonido doppler de la arteria femoral superficial y de la vena safena magna para tenerlos identificados durante la cirugía. B) Incisión longitudinal en cara lateral de muslo izquierdo, a través de la cual se realizó la evacuación del hematoma.

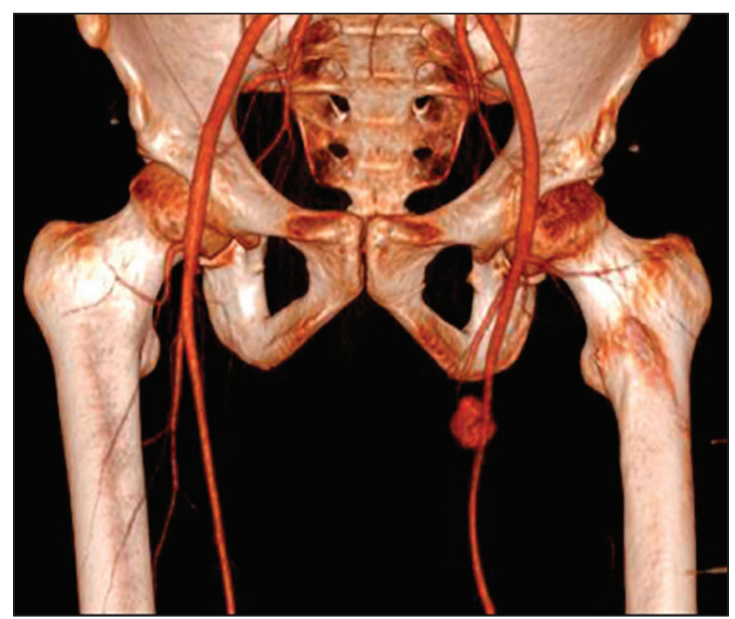

Figura 2. Pseudoaneurisma de la arteria femoral profunda con dimensiones de $2,3 \mathrm{~cm} \times 2,1 \mathrm{~cm} \times 2,7 \mathrm{~cm}$. punzocortante en cara lateral del tercio medio del muslo izquierdo en dirección medial. Recibe atención médica en hospital externo donde se le realiza cierre primario de herida. Al mes de evolución refiere aumento de volumen en el sitio de la lesión, el cual fue progresivo durante los próximos 4 meses, asociado a dolor y limitación del movimiento, llegando a ser incapacitante. Acude a valoración en otro nosocomio donde mediante ultrasonido doppler color de la extremidad afectada se reporta un pseudoaneurisma dependiente de la arteria femoral profunda de $3,5 \mathrm{~cm} \times 2 \mathrm{~cm} \times 2,3 \mathrm{~cm}$ en su tercio proximal y es referido a nuestra institución.

En la exploración física se observa el muslo izquierdo con aumento de volumen, sin cambios en la coloración, se palpa indurado, eutérmico, sin crepitantes, no fluctuante, sin frémito, con limitación en los arcos de movimiento; los pulsos pedio, tibial posterior, poplíteo y femoral se encuentran palpables, rítmicos y simétricos; llenado capilar inmediato y no se auscultan soplos. Se registra un índice tobillo brazo de 1 en ambas extremidades (Figura 1A). Se completa su abordaje con angiotomografía computarizada (angio TC), identificándose pseudoaneurisma de la arteria femoral profunda izquierda con dimensiones de $2,3 \mathrm{~cm} \times 2,1 \mathrm{~cm} \times 7 \mathrm{~cm}$ y hematoma adyacente de $13,5 \mathrm{~cm} \times 12,6 \mathrm{~cm} \times 23 \mathrm{~cm}$ con un volumen aproximado de $2000 \mathrm{cc}$ (Figuras 2 y 3 ). Se decide excluir el pseudoaneurisma vía endovascular colocando 8 coils, tipo $\mathrm{Azur}^{\mathbb{B}} \mathrm{HydroCoil}^{\mathbb{B}}$ con un diámetro del asa de $4 \mathrm{~mm}$ y una longitud de $15 \mathrm{~cm}$, en su interior con abordaje anterógrado para, posteriormente, evacuar el hematoma (Figura 4). A las $24 \mathrm{~h}$ posteriores se pasa a quirófano y se evacúa hematoma mediante una incisión longitudinal sobre la cara lateral del muslo izquierdo y se coloca un drenaje tipo Blake en el lecho quirúrgico (Figura 1B). Debido a buena evolución y mejoría de los síntomas, se egresa al paciente en su tercer día posoperatorio movilizándose por su cuenta, con cefalexina $500 \mathrm{mg}$ cada $8 \mathrm{~h}$ y analgesia. Se retira el drenaje a los 7 días de la cirugía con un gasto menor a $10 \mathrm{cc}$ en $24 \mathrm{~h}$ de características serohemáticas en su consulta de seguimiento.

\section{Discusión}

Los pseudoaneurismas de la arteria femoral profunda se presentan habitualmente como una masa dolorosa y pulsátil, síntoma que puede estar enmascarado por un hematoma o la formación de un trombo, como en el caso presentado. El diagnóstico se puede pasar por alto de manera temprana por la presencia de pulsos distales en la 
extremidad y la ausencia de síntomas complicando su diagnóstico, pero la presencia de datos como aumento de volumen, dolor, una masa pulsátil y anemia inexplicable, pueden sugerir la presencia de un pseudoaneurisma de la arteria femoral profunda. El tiempo que puede tardar en formarse es de 7 días a 13 semanas, situación que dificulta también su diagnóstico temprano ${ }^{3}$.

El diagnóstico de pseudoaneurisma se realiza mediante una buena historia clínica, una adecuada exploración física, un alto índice de sospecha y con el apoyo de estudios de imagen ${ }^{6}$. El ultrasonido doppler es el estudio de primera línea. En casos en los que este no sea concluyente o no logre caracterizar la lesión, la angioTC o la angiografía son los estudios de elección, además de que estos permiten planear la terapéutica. En el ultrasonido en ocasiones se observa el signo del yin-yang en el interior del pseudoaneurisma no trombosado, característico en este tipo de lesiones, el efecto arremolinado es producto del flujo anterógrado durante la sístole y el flujo retrógrado durante la diástole. En la angioTC también se observa este signo cuando el pseudoaneurisma está trombosado parcialmente. Se presenta como una masa redondeada, hiperdensa en una mitad, debido al contraste endovenoso e hipodensa en la otra mitad por la presencia del trombo ${ }^{4}$.

El manejo de elección continúa en debate, siendo aún el tratamiento convencional la cirugía abierta ${ }^{1}$. Los pseudoaneurismas menores a $3 \mathrm{~cm}$ o asintomáticos que no están en tratamiento con anticoagulación son propensos a que se trombosen de manera espontánea y se pueden vigilar por 4 semanas. El resto tienen una alta tasa de mortalidad, con morbilidad entre un 3 y $20 \%{ }^{5,6}$. Dentro de las opciones terapéuticas se encuentran: compresión del pseudoaneurisma, infiltración con trombina y exclusión endovascular ${ }^{6}$. Las características anatómicas son las que dictan el manejo de elección; el diámetro del cuello del aneurisma, la localización, ya sea distal o

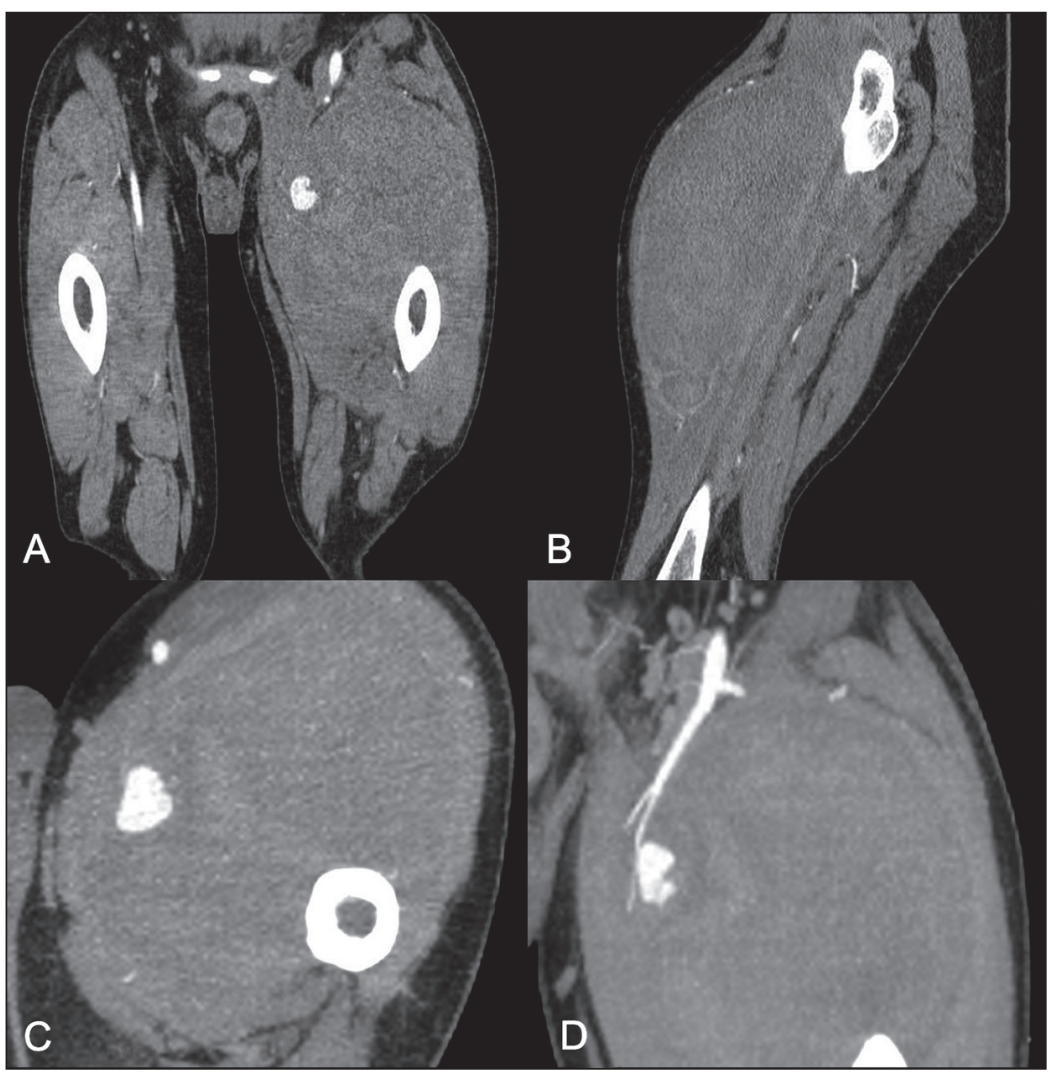

Figura 3. A) angioTC corte coronal; B) angioTC corte sagital; C) angioTC corte axial; D) se observa nacimiento de pseudoaneurisma dependiente de la arteria femoral profunda.

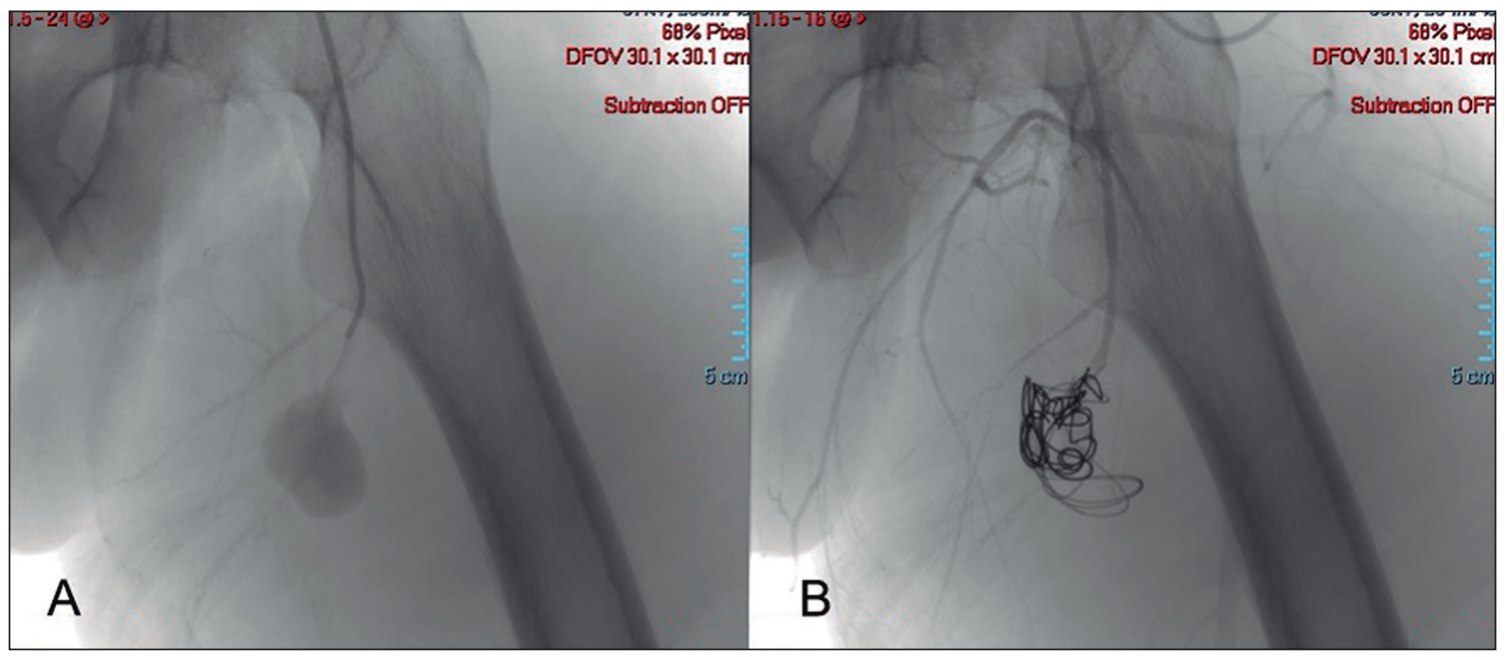

Figura 4. A) Se observa pseudoaneurisma de la arteria femoral profunda en angiografía; B) exclusión del pseudoaneurisma mediante la colocación de coils. 
proximal sobre la femoral profunda, las colaterales relevantes y las cirugías previas ${ }^{1}$.

Con el advenimiento de nuevas tecnologías y de técnicas menos invasivas se puede realizar como abordaje inicial una compresión guiada por ultrasonido en aquellos pacientes que no utilizan anticoagulantes y que el pseudoaneurisma es pequeño. La compresión puede durar hasta $1 \mathrm{~h}$ y los pacientes deben permanecer en cama con un vendaje compresivo por $8 \mathrm{~h}$, previo a su movilización, este manejo tiene una tasa de recurrencia de un $20 \%{ }^{5}$. Otras opciones terapéuticas son la inyección de trombina guiada por ultrasonido, la cual se administra de manera directa en el pseudoaneurisma en una concentración de $1.000 \mathrm{U} / \mathrm{ml}$, causando trombosis en 5 segundos, con una tasa de éxito de un $95 \%-98 \% \%^{2}$. La colocación de stents y la embolización con coils son terapéuticas adecuadas que permiten aislar el pseudoaneurisma sin afectar la circulación de colaterales, tiene una tasa elevada de éxito y disminuyen el riesgo de sangrado en la cirugía para drenar el hematoma adyacente $^{1}$.

\section{Conclusión}

En el caso presentado la exclusión del pseudoaneurisma mediante coils facilitó el control de éste y la evacuación del hematoma adyacente, disminuyendo el riesgo de sangrado. Por lo que consideramos adecuada esta conducta terapéutica en pseudoaneurismas de la femoral profunda.

\section{$\underline{\text { Responsabilidades éticas }}$}

Protección de personas y animales. Los autores declaran que para esta investigación no se han realizado experimentos en seres humanos ni en animales.

Confidencialidad de los datos. Los autores declaran que en este artículo no aparecen datos de pacientes.

Conflictos de interés: no hay.

\section{Bibliografía}

1. Pecoraro F, Dinoto E, Bracale UM, Badalamenti G, Farina A, Bajardi G. Symptomatic Deep Femoral Artery Pseudoaneurysm Endovascular Exclusion. Case Report and Literature Review. Ann Vasc Surg. 2017;42(April 2017):303. e5-303.e9. Disponible en: http://dx.doi. org/10.1016/j.avsg.2016.11.026.

2. Tisi P V, Callam MJ. Treatment for femoral pseudoaneurysms. Cochrane Database Syst Rev. 2013;(11):
CD004981. doi: 10.1002/14651858. CD004981.pub4.

3. Brunoro M, Baldassarre V, Sirignano P, Mansour W, Capoccia L, Speziale F. Endovascular Treatment of an Anterior Tibial Artery Pseudoaneurysm Secondary to Penetrating Trauma in a Young Patient: Case Report and Literature Review. Ann Vasc Surg. 2019;1-5. Disponible en: https://doi.org/10.1016/j. avsg.2019.03.005.

4. Badano F. Signo del Yin-Yang. Revista Argentina de Radiología
2010;74:403-5.

5. Saydam O, Serefli D, Engin AY, Atay M, Kunt A. Percutaneous Treatment of Deep Femoral Artery Pseudoaneurysm Due to Penetrating Trauma. Ann Vasc Dis. 2018;11:569-71.

6. Naouli H, Jiber H, Bouarhroum A. False Aneurysm of Perforating Branch of the Deep Femoral Artery - Report of Two Cases. Int J Surg Case Rep. 2015;14:369. doi: https://doi.org/10.1016/j. ijscr.2015.07.001. 